\title{
A recurrent neural network approach to quantitatively studying solar wind effects on TEC derived from GPS; preliminary results
}

\author{
J. B. Habarulema ${ }^{1,2}$, L.-A. McKinnell ${ }^{1,2}$, and B. D. L. Opperman ${ }^{1}$ \\ ${ }^{1}$ Hermanus Magnetic Observatory, 7200 Hermanus, South Africa \\ ${ }^{2}$ Department of Physics and Electronics, Rhodes University, 6140, Grahamstown, South Africa
}

Received: 23 June 2008 - Revised: 16 March 2009 - Accepted: 29 April 2009 - Published: 7 May 2009

\begin{abstract}
This paper attempts to describe the search for the parameter(s) to represent solar wind effects in Global Positioning System total electron content (GPS TEC) modelling using the technique of neural networks (NNs). A study is carried out by including solar wind velocity $\left(V_{s w}\right)$, proton number density $\left(N_{p}\right)$ and the $B_{z}$ component of the interplanetary magnetic field (IMF $B_{z}$ ) obtained from the Advanced Composition Explorer (ACE) satellite as separate inputs to the NN each along with day number of the year (DN), hour (HR), a 4-month running mean of the daily sunspot number (R4) and the running mean of the previous eight 3-hourly magnetic A index values (A8). Hourly GPS TEC values derived from a dual frequency receiver located at Sutherland $\left(32.38^{\circ} \mathrm{S}, 20.81^{\circ} \mathrm{E}\right)$, South Africa for 8 years (2000-2007) have been used to train the Elman neural network (ENN) and the result has been used to predict TEC variations for a GPS station located at Cape Town $\left(33.95^{\circ} \mathrm{S}, 18.47^{\circ} \mathrm{E}\right)$. Quantitative results indicate that each of the parameters considered may have some degree of influence on GPS TEC at certain periods although a decrease in prediction accuracy is also observed for some parameters for different days and seasons. It is also evident that there is still a difficulty in predicting TEC values during disturbed conditions. The improvements and degradation in prediction accuracies are both close to the benchmark values which lends weight to the belief that diurnal, seasonal, solar and magnetic variabilities may be the major determinants of TEC variability.
\end{abstract}

Keywords. Ionosphere (Mid-latitude ionosphere; Modeling and forecasting; Instruments and techniques)

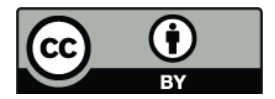

Correspondence to: J. B. Habarulema (jhabarulema@hmo.ac.za)

\section{Introduction}

The solar wind-magnetosphere-ionosphere (SW-MAG-ION) system is not an easy phenomenon to breakdown in order to deal with the respective physical mechanisms responsible for the physics and chemistry describing the behaviour of different constituent ions and molecules that contribute to the changes of ionospheric parameters. According to Lyon (2000), the SW-MAG-ION form a single system that owes its existence to the energy and momentum transfer from the solar wind to the ionosphere through the magnetosphere. Among the ionospheric parameters influenced by this transfer of energy is the total electron content (TEC) especially during geomagnetic storms. One of the first experimental evidence between the solar wind and variations in electron content was reported by Sethia et al. (1978) where an inverse relationship between plasmaspheric TEC and solar wind velocity was observed during the pre-storm and storm conditions. A more recent study pointed out that separating agents responsible for ionospheric disturbances especially during magnetically disturbed conditions is complicated due to the complexity of the correlation between the solar wind dynamics and ionospheric variations (Biktash et al., 2008). Biktash et al. (2008) showed that the solar wind plays an important role in controlling the equatorial ionosphere during geomagnetic storms by explaining the IMF $B_{z}$ effects on ionospheric parameters. While most works considered the study of ionospheric behaviour with respect to solar wind dynamics before, during and after storm conditions (e.g. Meza et al., 2005; Fedrizzi et al., 2005; Biktash et al., 2008; Li et al., 2008), this paper attempts to find a quantitative relationship between solar wind and TEC variability through modelling and taking into account both quiet and disturbed circumstances over a relatively long period of time. Like other ionospheric parameters, TEC varies nonlinearly and this makes quantifying its fluctuations through analytical algorithms a complicated task and therefore it is

Published by Copernicus Publications on behalf of the European Geosciences Union. 


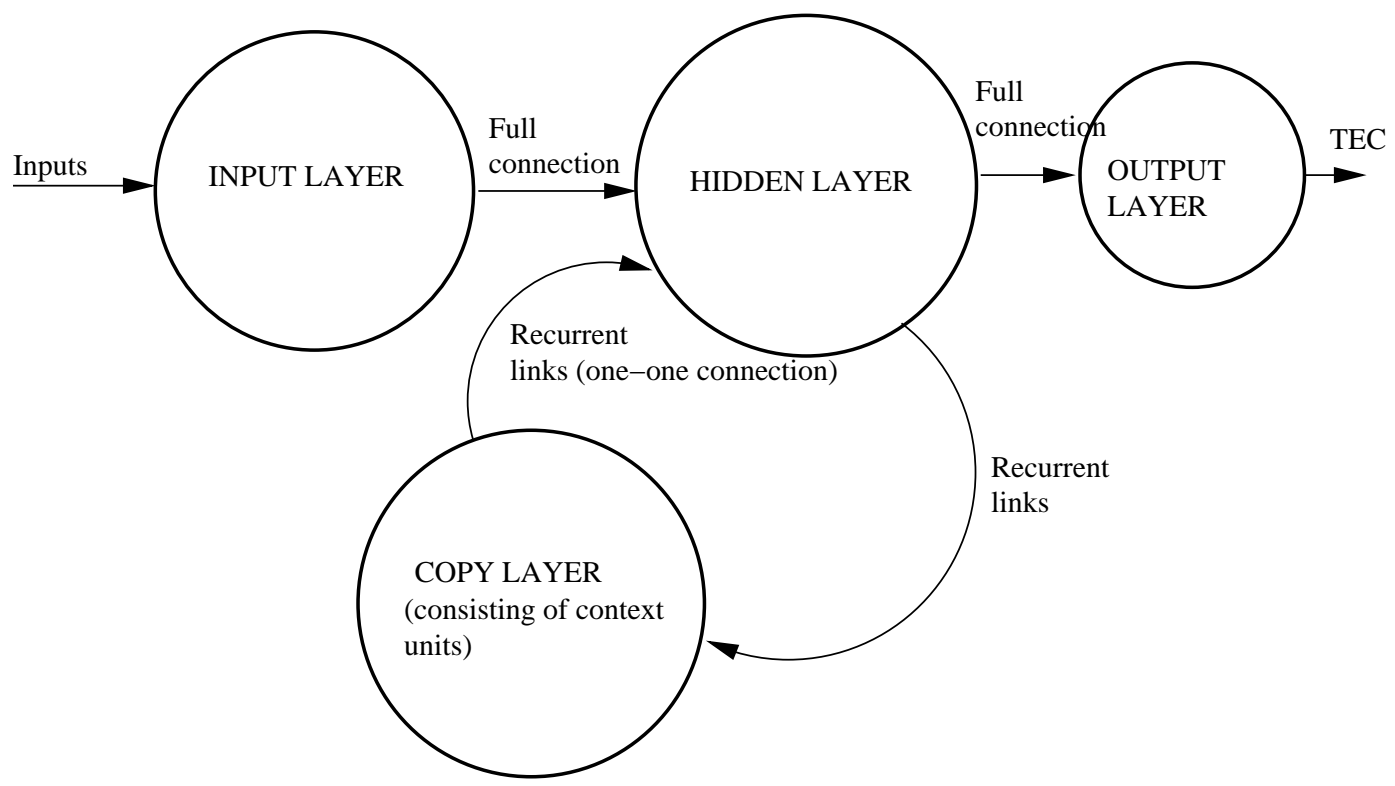

Fig. 1. An Elman neural network structure having one input, one hidden and one output layers.
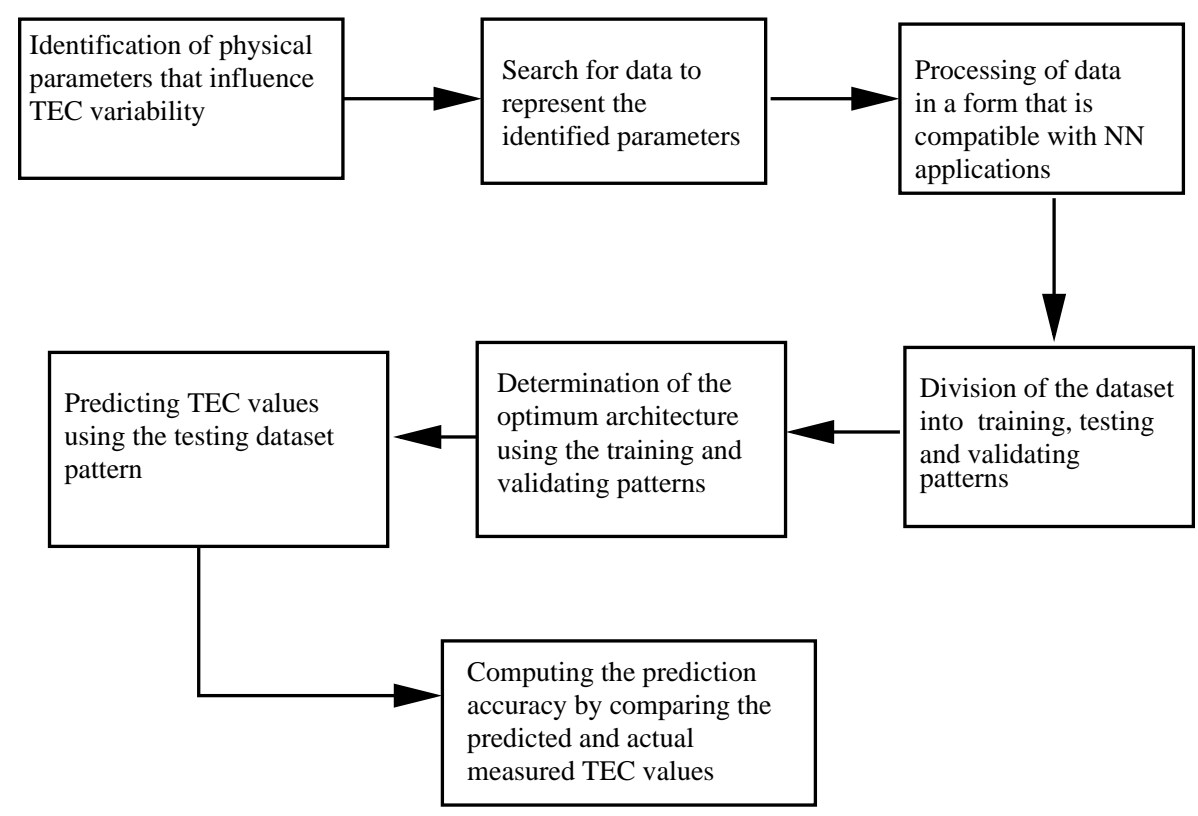

Fig. 2. A flow diagram illustrating the procedure followed during the modelling of TEC undertaken in this study.

preferable to resort to empirical modelling. In this regard, given enough historic data describing the variational pattern of a certain physical quantity, neural networks (NNs) have proved to be relatively efficient in estimations or predictions involving non-linear approximations particularly TEC in terms of physical and geophysical parameters (HernandezPajares et al., 1997; Tulunay et al., 2006; Habarulema et al., 2007, 2009). The understanding of TEC variability, its fore- casting and now casting is very beneficial for applications involving long range communications, GPS surveying, navigation and other space weather related activities (Stankov et al., 2001; Tulunay et al., 2006). According to the knowledge of the authors, no published work is currently available so far that quantifies the solar wind effects on GPS TEC over the African region. The attempt undertaken by Habarulema (2007) on a small database over the Square Kilometer Array 

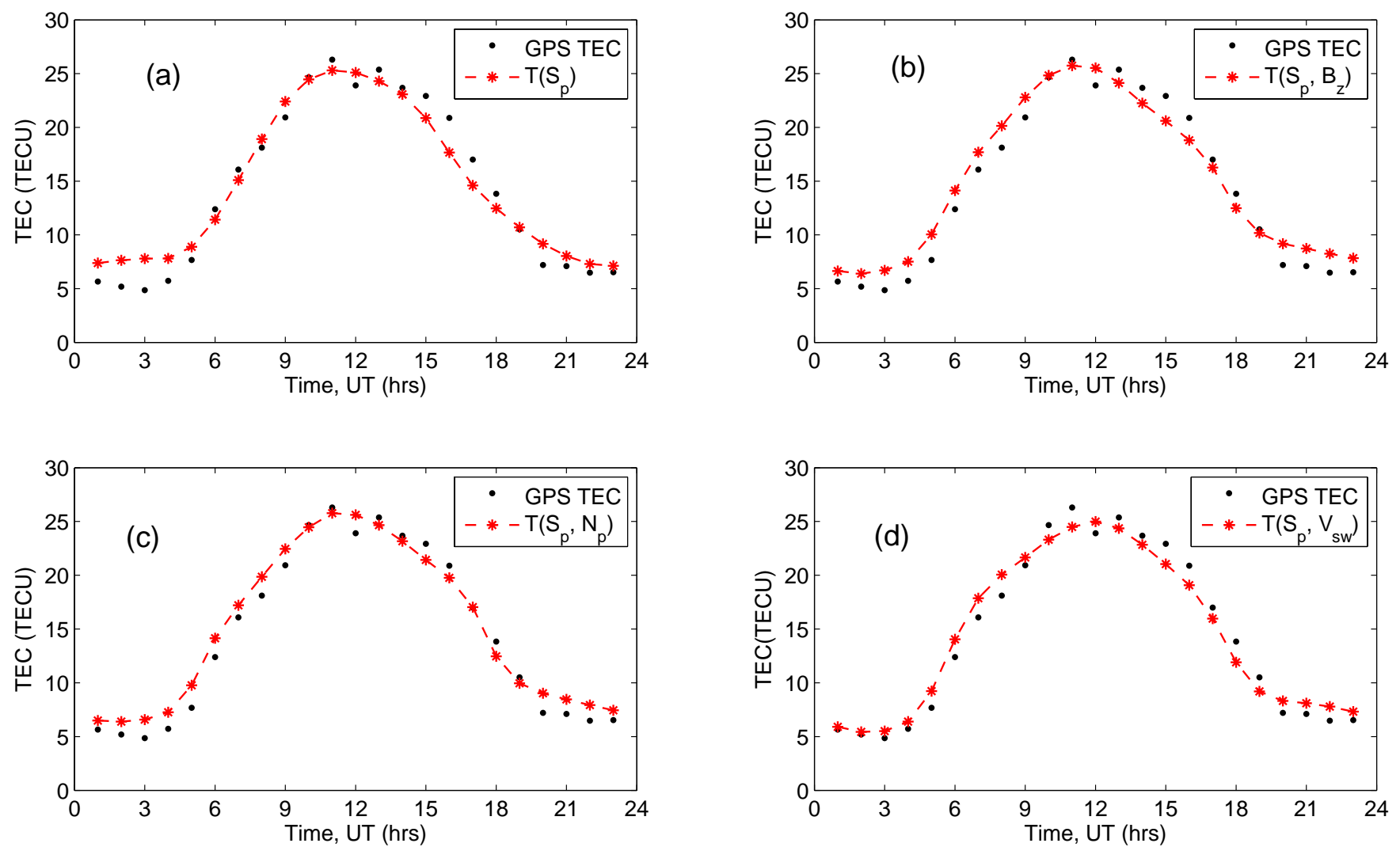

Fig. 3. Comparison between hourly GPS TEC and the corresponding predictions for (a) $T\left(S_{p}\right)$, (b) $T\left(S_{p}, B_{z}\right),(\mathbf{c}) T\left(S_{p}, N_{p}\right)$ and (d) $T\left(S_{p}\right.$, $V_{s w}$ ); for day 58 in 2005 .

(SKA) Hub location $\left(30.71^{\circ} \mathrm{S}, 21.39^{\circ} \mathrm{E}\right)$, South Africa at 10:00 UT showed that a parameter representing solar wind could be an additional input for the NN to learn and generalise TEC patterns. Habarulema (2007) considered $V_{s w}$ and $N_{p}$ (since electron number density was not available at that time) as separate inputs to the $\mathrm{NN}$ and quantification results using the root mean square error (RMSE) method were generally inconclusive. This work is a more comprehensive extension in a sense that it also quantitatively investigates the variations of IMF $B_{z}$ with TEC fluctuations. Space weather forecasts and correlation studies between solar wind, magnetosphere and geomagnetic field measurements have been performed with NNs (Wu and Lundstedt, 1997; Weigel et al., 2002, 2003; Vandegriff et al., 2005; Lundestedt, 2006). Vandegriff et al. (2005) used NNs to demonstrate that they were capable of predicting the arrival of interplanetary shocks on the Earth. Weigel et al. $(2002,2003)$ also used NNs to map solar wind measurements to the changes in ground magnetic field levels and their predictability with time. These authors demonstrated that the solar wind and rate of change of geomagnetic field are related and have a strong dependance on local time and latitude. Enough literature, the underlying principles involved and assumptions made about the derivation of TEC from GPS data are available (e.g. Langley, 2000;
Fedrizzi et al., 2005) and will not be repeated here. It is however worth mentioning that the TEC data used in this study were derived using the Adjusted Spherical Harmonic Analysis (ASHA) algorithm and its details can be found in Opperman et al. (2007) and Opperman (2007). This paper presents results obtained by predicting GPS TEC as a function of seasonal variation, diurnal variation, magnetic activity, solar activity and the parameters representing the solar wind. GPS TEC values predicted using the first four parameters act as a benchmark while determining the solar wind parameter effects on TEC. The seasonal and diurnal variations are represented by day number (DN) and hour (HR), respectively. The 4-month running mean of daily sunspot number (R4) represents solar activity while the magnetic activity is represented by the running mean of the previous eight 3-hourly magnetic A index values (A8). In this paper, the function $T\left(S_{p}\right)$ is defined to represent predicted TEC as a function of DN, HR, A8 and R4 i.e. $T\left(S_{p}\right)=\mathrm{T}(\mathrm{DN}, \mathrm{HR}, \mathrm{A} 8, \mathrm{R} 4)$ so that $T\left(S_{p}, B_{z}\right), T\left(S_{p}, V_{s w}\right)$ and $T\left(S_{p}, N_{p}\right)$ represent predicted TEC values after separately adding $B_{z}, V_{s w}, N_{p}$ respectively to $S_{p}$. The prediction was undertaken for prestorm, storm and post storm days to assess the performance of the $\mathrm{NN}$ model during geomagnetic disturbed conditions (6-10 and 14-18 May 2005). The overall discussion in this paper tried 

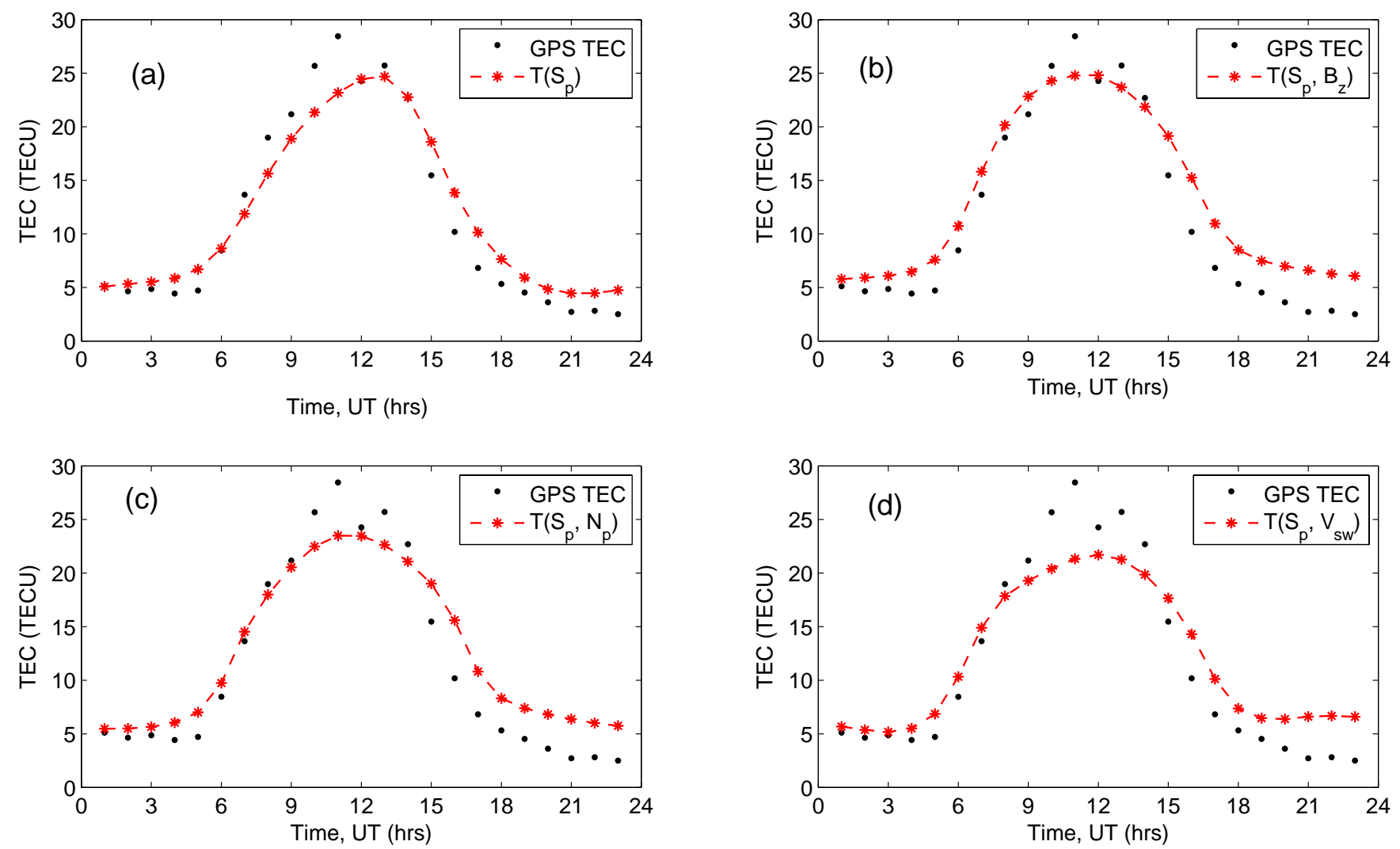

Fig. 4. Similar to Fig. 3, for day 104 in 2005.

to answer the question "If the solar wind has an influence on TEC fluctuations, should it be taken into account along with other standard parameters during TEC predictability or modelling?"

\section{Data sources}

The data used for the $V_{s w}, N_{p}$ and the IMF $B_{z}$ parameter recorded by the Advanced Composition Explorer (ACE) satellite were downloaded from http://cdaweb.gsfc.nasa.gov/ cdaweb/sp_phys/. GPS TEC data were derived from the dual frequency receivers located at Sutherland $\left(32.38^{\circ} \mathrm{S}\right.$, $\left.20.81^{\circ} \mathrm{E}\right)$ and Cape Town $\left(33.95^{\circ} \mathrm{S}, 18.47^{\circ} \mathrm{E}\right)$ using the ASHA algorithm which makes use of the mapping function that assumes the ionosphere to be a single layer of height $350 \mathrm{~km}$ (Opperman et al., 2007). The availability of GPS TEC data was taken as a reference point for the consideration of solar wind data. The database contained periods with GPS TEC data but without either $V_{s w}, N_{p}$ or IMF $B_{z}$ data. This discontinuity in the ACE data was catered for, by replacing these days with the monthly mean values of days for which data was available according to the following equation:

$M_{d}=\frac{1}{N_{d}} \sum_{i=1}^{N_{d}} X_{j=1,2,3}$ where $M_{d}$ represents missing data in $V_{s w}\left(X_{j=1}\right), N_{p}\left(X_{j=2}\right)$ and $B_{z}\left(X_{j=3}\right)$,

$i=1,2, \ldots, N_{d}$, with $\mathrm{N}_{d}$ being the total number of days for a given month.

It should be noted that the most significant amount of missing data was found in the $N_{p}$ parameter followed by $V_{s w}$ and finally $B_{z}$. The ACE satellite is roughly located at the L1 point (a point where the gravitational attraction on the satellite from the Sun and the Earth cancel out), which is at a distance of more than $200 R_{E}\left(R_{E}\right.$ is the Earth's radius in $\left.\mathrm{km}\right)$ from the Earth's surface, making it relatively more steady with respect to both the Sun and the Earth. The communication time between the ACE satellite and the Earth is assumed to be small and is hence ignored (Weigel et al., 2002, 2003) during the ENN modelling.

\section{Recurrent networks, data processing and NN training}

\subsection{Recurrent networks}

A $\mathrm{NN}$ is a powerful tool that can be used to perform the tasks of learning and generalising the variational behaviours and patterns of parameters that exhibit non-linear characteristics through the input-output mapping process. NNs have 

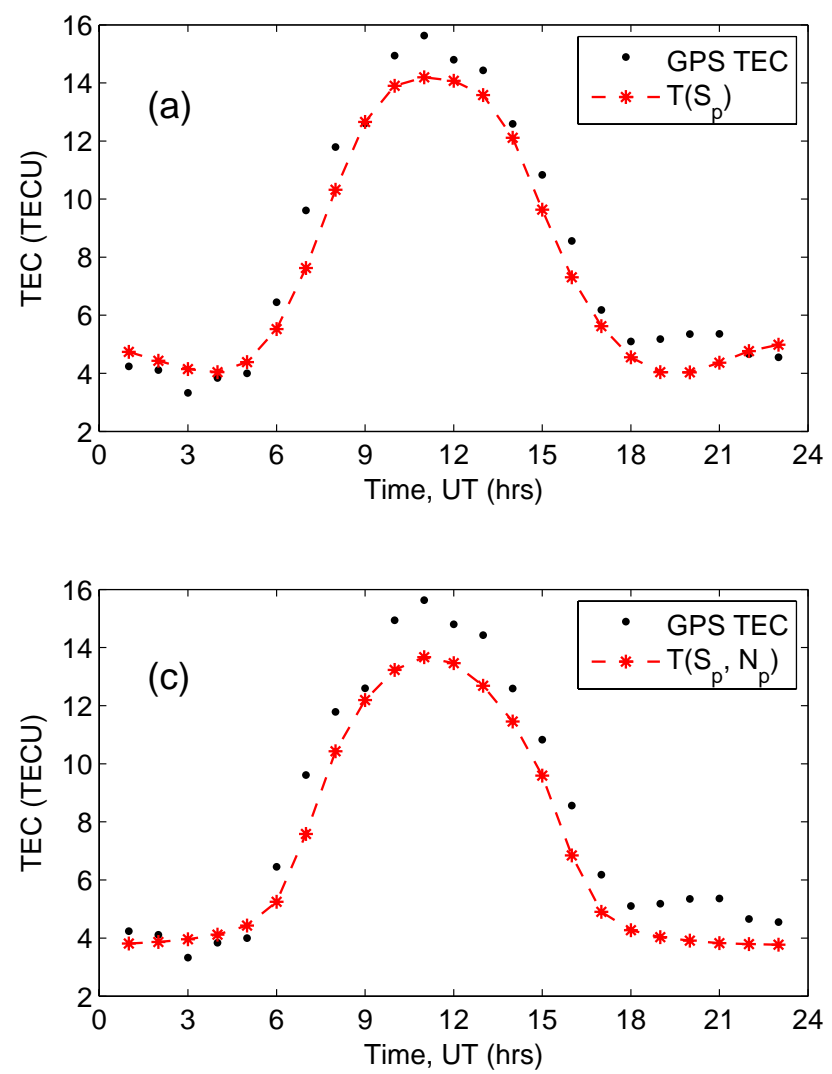

Fig. 5. Similar to Fig. 3, for day 185 in 2005.

been widely used in many applications that deal with nonlinear approximations. A number of textbooks (e.g. Bishop, 1995; Haykin, 1994; Fausett, 1994) and published papers (e.g. Weigel et al., 2002; Marra and Marabito, 2005; Tulunay et al., 2006; Vandegriff et al., 2005; Habarulema et al., 2007 , etc.) elaborate the effectiveness and procedure of using this technique in empirical modelling. There are various ways and algorithms available for implementing NNs (Haykin, 1994; Bishop, 1995). This work uses one form of recurrent networks known as Elman networks which handles function learning based on a combination of current input parameters at any time and a series of previously learned parameters with the corresponding outputs of the entire network connection (Elman, 1990). One of the major differences between ordinary feed forward NNs and recurrent Elman neural networks (ENNs) is that the latter has a copy layer of the hidden neurons (Haykin, 1994). The functional approach of ENNs enables the correction of time delay between the various input parameters and the output parameter during $\mathrm{NN}$ training and validating processes. In simple terms, an ENN is like a modified feed forward NN since it has an additional layer consisting of context units which allows hidden unit patterns to be fed back to themselves (Elman, 1990) and uses a back propagation algorithm. In the ENN, each hidden unit has a corresponding context unit and
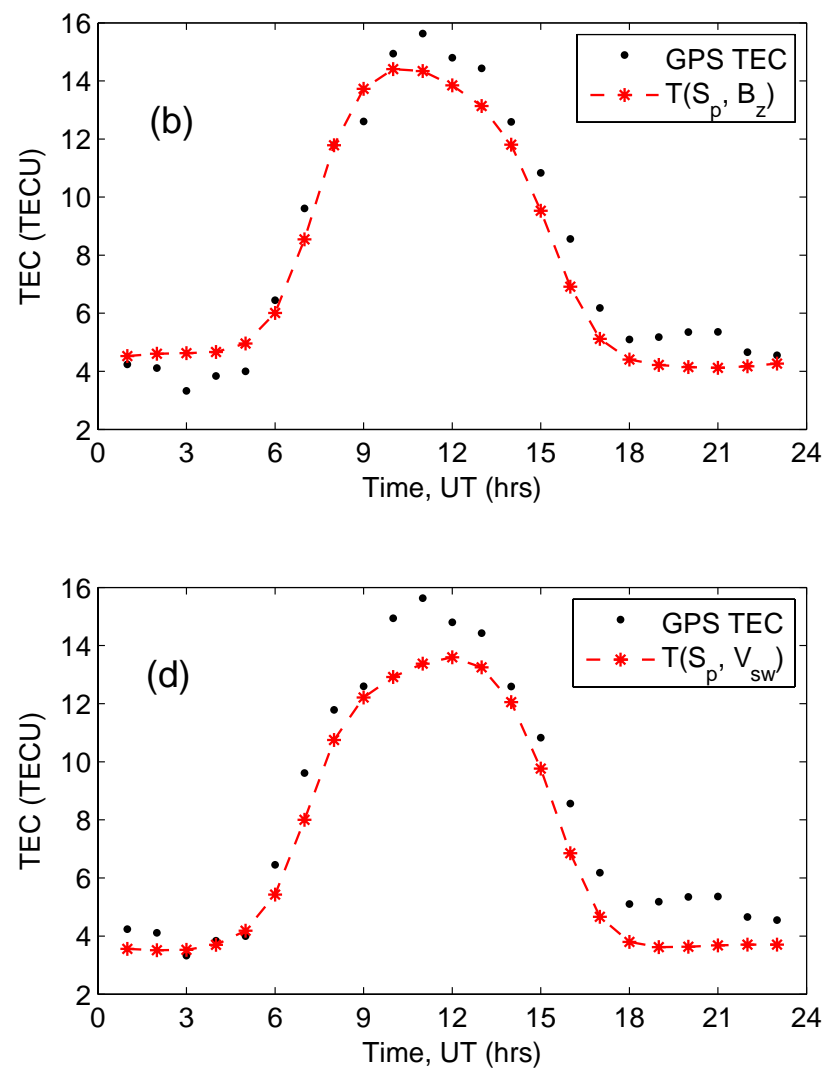

therefore the number of hidden neurons or nodes is equal to the number of context units in the copy layer (Marra and Marabito, 2005). Figure 1 shows an example of a simplified structure of the ENN having one input, hidden and output layers. The mathematical computations are not represented for clarity and simplicity. The output for single hidden layered ENN, with $n$ input units, $m$ hidden units and one output unit can be expressed in terms of the activation function and asociated weights along with the corresponding biases at different times in an iterative process (Wu and Lundstedt, 1997; Bodén, 2002) as follows;

$$
\begin{array}{r}
X_{j}(t)=f\left(\sum_{k=1}^{n} v_{j k} x_{k}(t)+\sum_{h=1}^{m} u_{j h} y_{h}(t-1)+b_{j}\right) \\
X_{o}(t)=\sum_{j=1}^{m} X_{j}(t) w_{o j}+b_{o}
\end{array}
$$

where $X_{j}(t)$ is the output of the hidden unit $j$ at time $t$, $f=1 /\left(1+e^{-x}\right)$ is the activation function, $x$ is the input training vector, $j$ and $h$ are indices for the hidden and context nodes, $k$ and $o$ are indices for input and output nodes respectively, $X_{o}(t)$ is the output of the output unit $o$ at time $t, v, u$ and $w$ are the connection weights associated with the input, hidden and output layers, respectively and $b$ is the bias. For a 

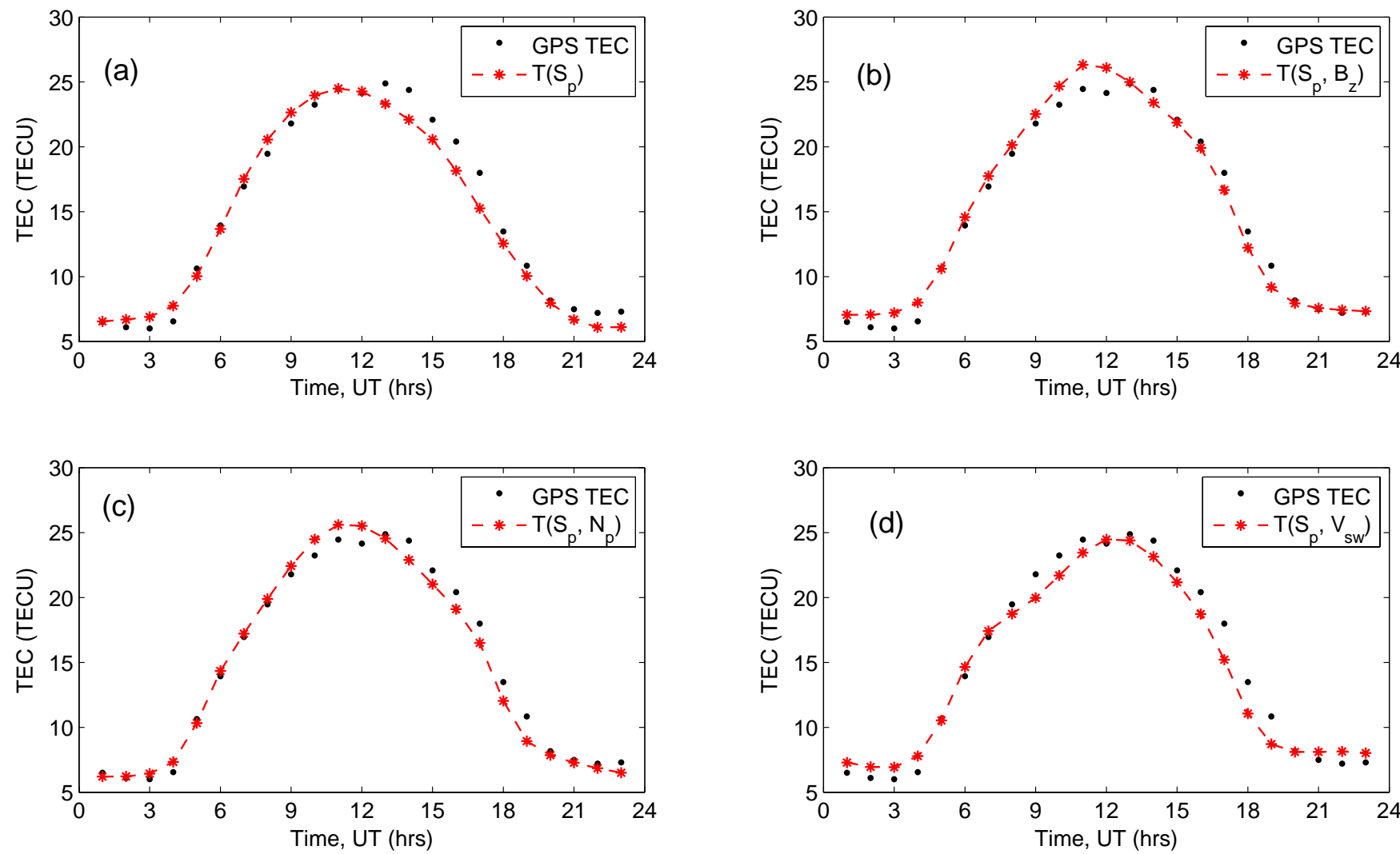

Fig. 6. Similar to Fig. 3, for day 285 in 2005.

detailed description about ENN, the reader is referred to the references within this section.

\subsection{Data processing and NN training}

Data for the identified parameters were processed and organised in a form that is compatible with NNs. During the data organisation process, the day number (DN) and hour (HR) were each split into sine and cosine components to allow for the continuous trend of data (Poole and McKinnell, 2000) as follows;

$$
\begin{array}{cc}
\mathrm{DNS}=\sin \left(\frac{2 \pi \times \mathrm{DN}}{365.25}\right) & \mathrm{DNC}=\cos \left(\frac{2 \pi \times \mathrm{DN}}{365.25}\right) \\
\mathrm{HRS}=\sin \left(\frac{2 \pi \times \mathrm{HR}}{24}\right) & \mathrm{HRC}=\cos \left(\frac{2 \pi \times \mathrm{HR}}{24}\right)
\end{array}
$$

where DNS, DNC, HRS and HRC are the sine and cosine components of DN and HR, respectively.

The procedure for determining magnetic and solar activity representations is clearly presented in Habarulema et al. (2007). Hourly GPS TEC values were extracted using the ASHA algorithm from the GPS observations. A total of 50228 data points were used in $\mathrm{NN}$ training and validating processes each comprising of DNC, DNS, HRC, HRS, A8, R4 and either $V_{s w}, N_{p}$ or $B_{z}$. The ENN uses six or seven input parameters and one output parameter (TEC) through out the entire study. During the implementation of the NN technique, data for 2000-2007 was used in training and validating, while the 2005 dataset was used for verifying the accuracy of the NN model. Figure 2 shows a simplied flow chart of the entire process. This study was conducted in two phases. The first one involved the prediction of TEC as a function of diurnal variation, seasonal variation, solar activity and magnetic activity, which acted as a benchmark while quantifying the solar wind effects on GPS TEC. After determining the optimum NN architecture and computing the prediction accuracy, each of the solar wind parameters was included as a separate input to the $\mathrm{NN}$ along with $\mathrm{DN}, \mathrm{HR}$, A8, R4 and the procedure repeated. Details of data analysis are presented in Sect. 5 .

\section{The solar wind and TEC variations}

Solar wind is a stream of energetic particles (in the form of a plasma) that is continuously ejected from the sun and reaches the Earth's atmosphere at a speed typically in the range $300 \mathrm{~km} / \mathrm{s}$ to $700 \mathrm{~km} / \mathrm{s}$. Its composition is mainly electrons and protons (about 95\%). The variations of solar wind dynamics with TEC are more significantly observed during 

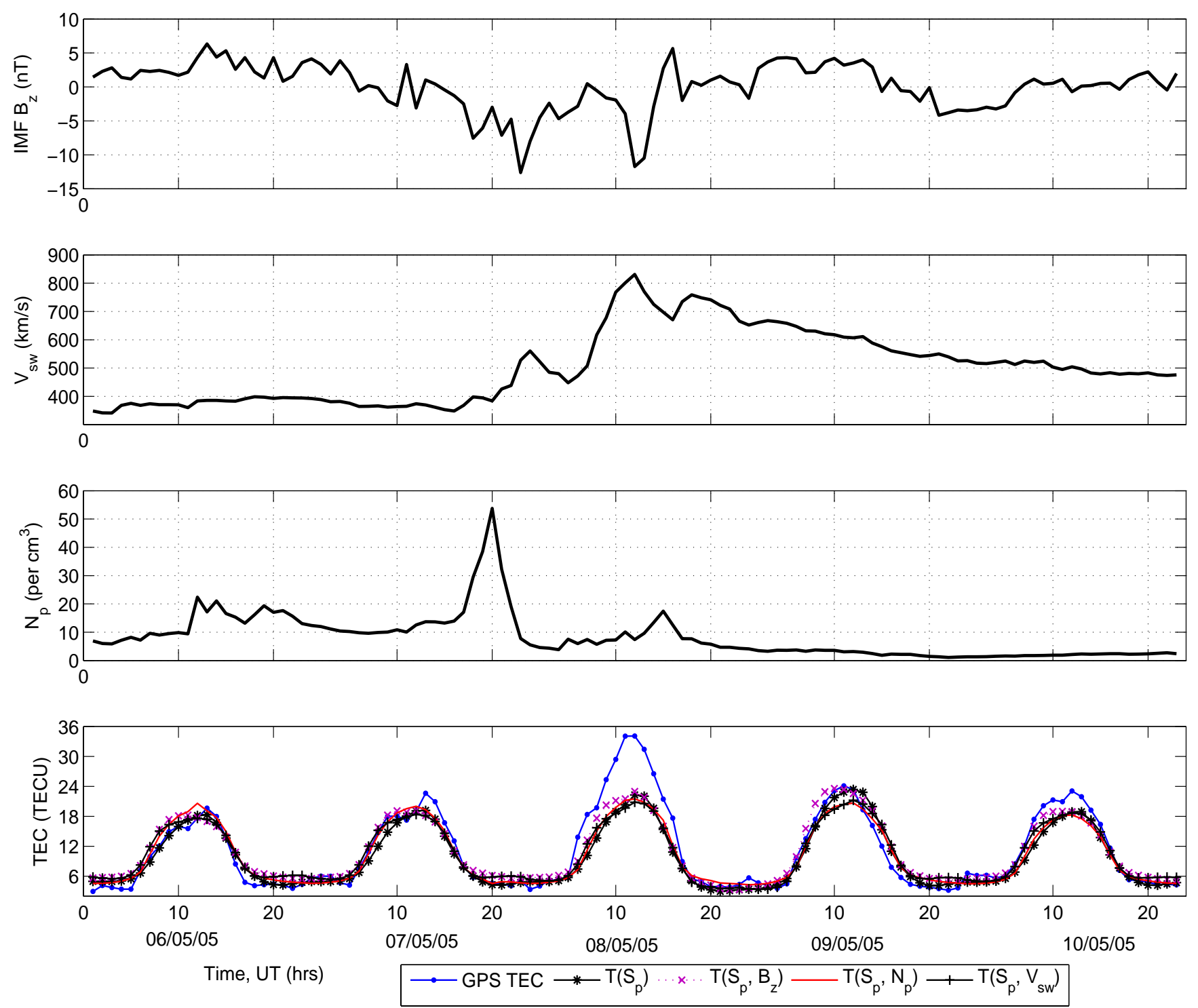

Fig. 7. Variations of the considered solar wind parameters and TEC values (derived and predicted) during 6-10 May 2005.

magnetic storms. Jakowski et al. (1999) studied the relationship between the solar wind and TEC before and around solar minimum at different European latitudes using $V_{s w}$ as one of the parameters and found that there exists an anticorrelation. The observed delay between $V_{s w}$ and TEC was interpreted as an indication of close coupling of the solar wind with the ionosphere-thermosphere dynamics related to the observed negative phases of ionospheric storms a few days after storm onset. Additionally, various studies at all latitudes have shown that TEC is influenced during geomagnetic storms, a process believed to be driven by injection of the solar wind energy into the magnetosphere and characterised by the south ward turning of the IMF $B_{z}$ (Meza et al., 2005; Fedrizzi et al., 2005; Biktash et al., 2008). In this work, the idea was to include $V_{s w}$, IMF $B_{z}$ and the electron number density $\left(N_{e}\right)$ to the NN. However, data for $N_{e}$ was not available and since the number of electrons in the solar wind is roughly equal to the number of protons, $N_{p}$ was used instead. The solar wind parameter is considered in this empirical modelling with the prospect of capturing plasmapheric TEC variations during magnetic storms especially at high altitudes. However, a very recent study pointed out that separating agents forming ionospheric disturbances during storm conditions is too difficult due to the complexity of the correlation between solar wind dynamics and the ionospheric variations (Biktash et al., 2008). A decrease of TEC (from both GPS and ionosonde) has been observed over South Africa during the main phases of geomagnetic storms. During this period, an increase in $V_{s w}$ and $N_{p}$ is observed. Sethia et al. (1978) showed that there is an inverse relationship between plasmaspheric TEC 

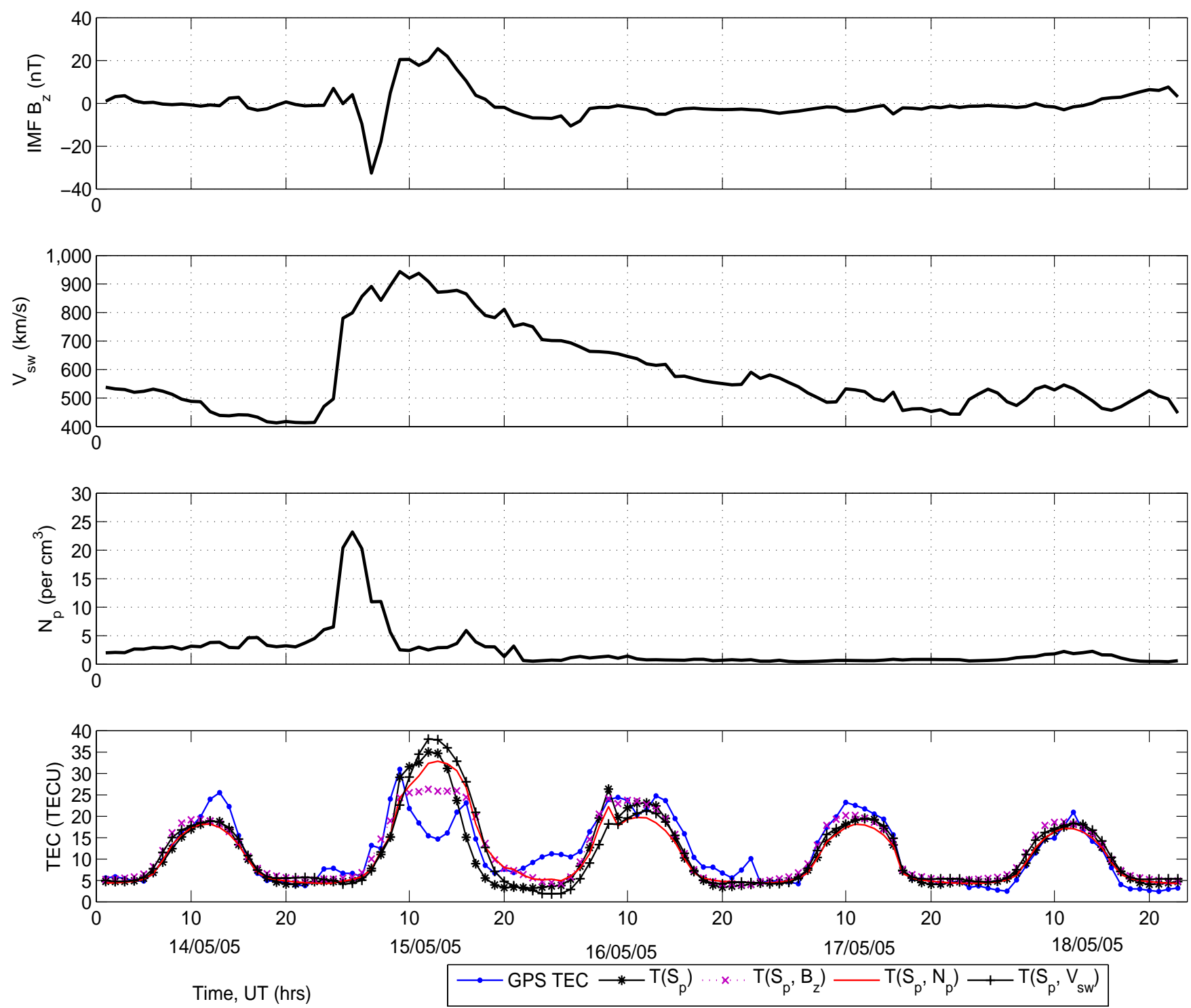

Fig. 8. Variations of the considered solar wind parameters and TEC values (derived and predicted) during 14-18 May 2005.

and $V_{s w}$ over an equatorial station, Ootacamund $\left(11.4^{\circ} \mathrm{N}\right.$, $76.6^{\circ} \mathrm{E}$ ) as the first experimental evidence during pre-storm and storm conditions. These authors utilised electron content measurements made by Faraday rotation and group delay techniques using radio beacon signals from the Application Technology Satellite (ATS-6) to compute the electron content of the plasmasphere. Contrary to studies that deal with TEC and solar wind variations during storm conditions, this study presents modelling efforts undertaken using data for both quiet and disturbed conditions to try and quantitatively determine effects of solar wind on TEC fluctuations. In this work, the $V_{s w}$ magnitude is computed by combining the three components of velocity in $\mathrm{x}-, \mathrm{y}$ - and $\mathrm{z}$-directions using the following equation:
$V_{s w}=\sqrt{V_{x}^{2}+V_{y}^{2}+V_{z}^{2}}$

where $V_{s w}$ is the magnitude of solar wind velocity.

The IMF $B_{z}$ is one of the indicators usually used to study geomagnetically disturbed conditions on solar-terrestrial systems. The physical mechanisms guiding the outcomes of magnetic storms on TEC variations are different for different latitudes and sometimes seasons. It has been observed that negative storm effects are the most likely outcomes over midlatitudes although positive storm effects are also sometimes evident (Buonsanto, 1999). Significant variabilities of the considered solar wind parameters and TEC are observed during the considered magnetic storms of May 2005 as shown in Figs. 7 and 8, Sect. 6. 

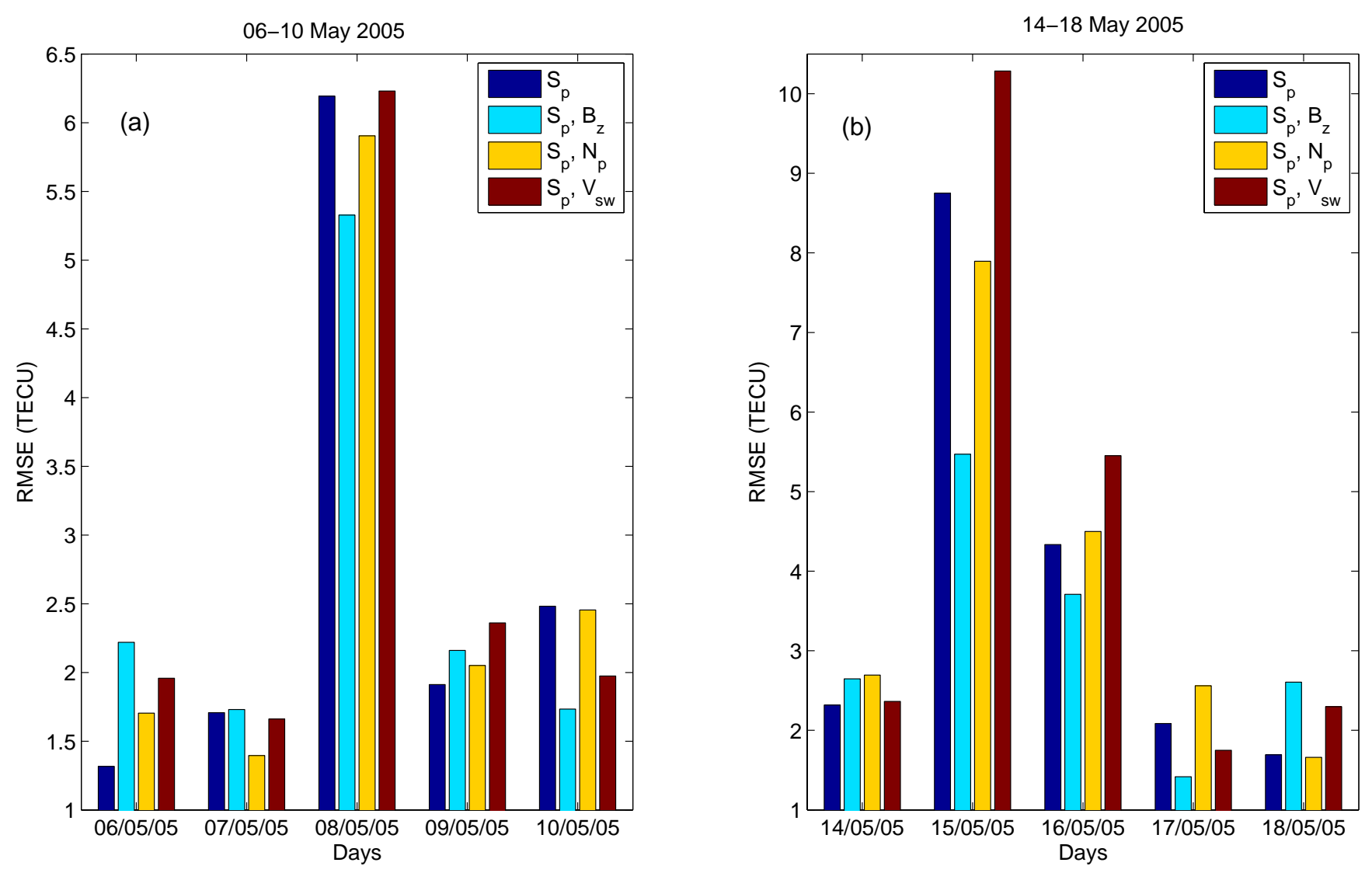

Fig. 9. Computed RMSE values between GPS TEC and the predicted TEC as a function of all combined parameters considered, for 6-10 and 14-18 May 2005.

\section{Data analysis}

As mentioned in Sect. 2, hourly GPS TEC data for the two GPS sites (equiped with Ashtech geodetic grade dual frequency receivers) were estimated using the ASHA algorithm. It is known that TEC variability is influenced by seasonal and diurnal variations, solar and magnetic activities and the geographic position of the GPS receiver (Hofmann-Wellenhof et al., 1992). Only the first four parameters were considered in this work since we dealt with single station studies. It is clear that a NN would not learn any thing from TEC variability with respect to a constant geographical position. The dataset under consideration consisting of DN, HR, R4, A8 and the solar wind parameters were divided into training and validating patterns in the ratio of 7:3, respectively. Hourly data for 2000-2007 over Sutherland were used for the training and validating processes while hourly data for 2005 over Cape Town were used for verifying. It is important to note that the verification dataset was not included in training, but fell within the training period thus taking into account the solar activity level changes. It has been previously shown that a NN model developed at a particular GPS station can be used to predict TEC variability over another GPS receiver station that was not involved in NN model development. This has demonstrated the temporal and to some extent spatial capability of the NN in dealing with such non-linear approximations within a particular latitudinal range (Habarulema et al., 2009). The ability of the NN model to capture TEC variability at a different station with data not included in training may be attributed to the fact that since the stations have a latitudinal difference of $\sim 2^{\circ}$, the ionospheric pierce point (IPP) which provides an input for the GPS vertical TEC on the receiver used in the NN training (Sutherland) may cover the geographical location of the receiver located at Cape Town. Keeping DN, HR, A8 and R4 constant, the solar wind parameter representation $\left(V_{s w}, N_{p}\right.$ or $\left.B_{z}\right)$ was each included separately in the ENN training and the respective networks verified. With effect to this, a number of recurrent ENNs were trained in a search for the optimum architecture. Training was monitored until the mean square errors on the testing dataset decreased (Habarulema et al., 2007). There is no direct way of determining the number of hidden nodes in the hidden layer. Increasing the number of hidden layers was found not to have a significant change on the accuracy of results, but makes training easier in terms of time in some cases (Haykin, 1994). An optimum architecture for this work 

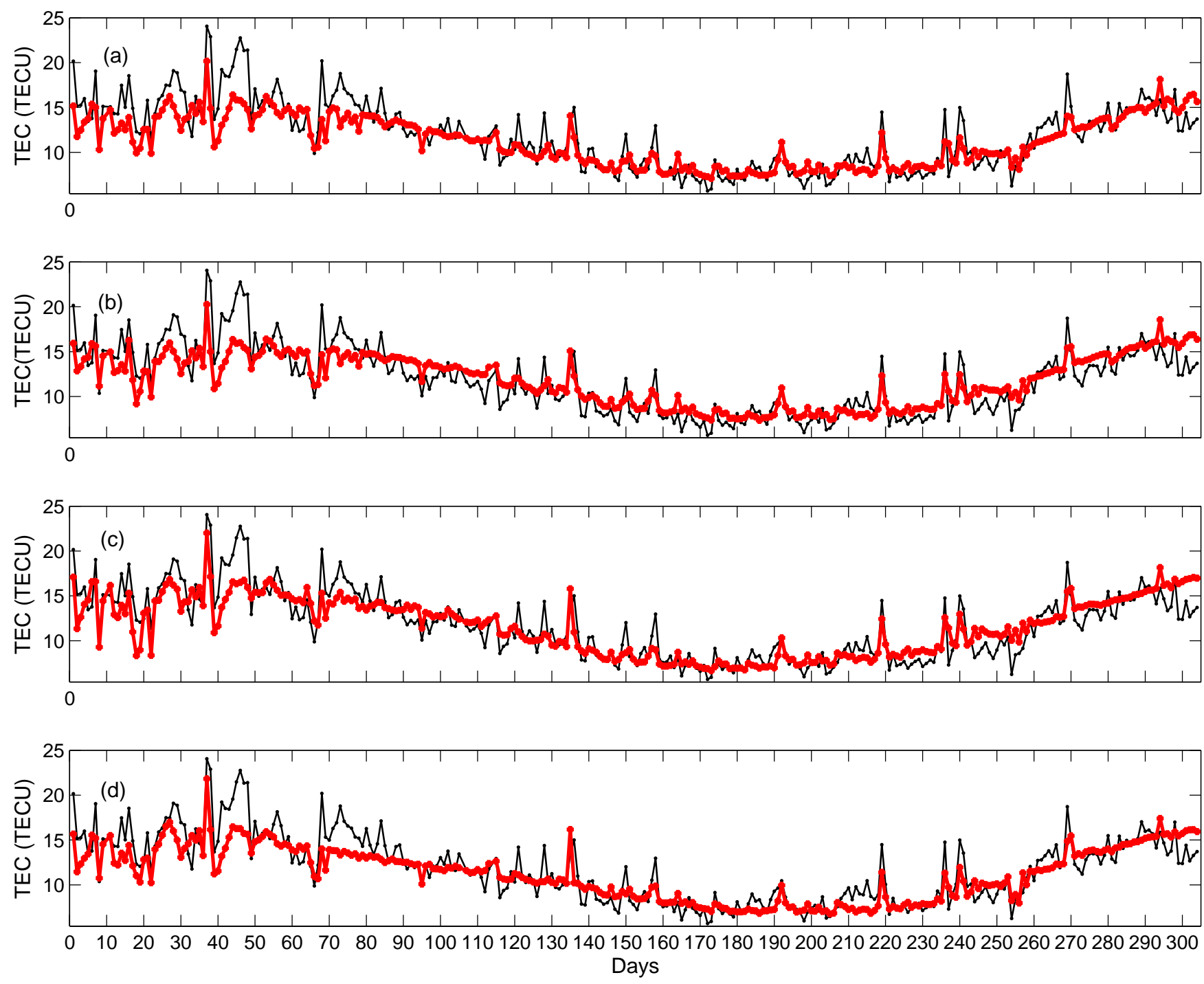

Fig. 10. Comparison between average daily GPS TEC (thin black line) and the corresponding predictions (thick red line); (a) $T\left(S_{p}\right)$, (b) $T\left(S_{p}, B_{z}\right)$, (c) $T\left(S_{p}, N_{p}\right)$ and (d) $T\left(S_{p}, V_{s w}\right)$; for the verifying year 2005 .

consisted of the configuration 6:8:1 for the standard parameters ( $S_{p}=\mathrm{DN}, \mathrm{HR}, \mathrm{A} 8$ and R4) and changed to 7:9:1 when the solar wind parameter was included in the study. The architecture consisted of one input layer, one hidden layer and one output layer having one neuron. In the determination of the optimum NN architecture, the root mean square error (RMSE) method has been used. The followed procedure was the addition of one hidden node at a time, training the NN, testing it with data and finally computing the RMSE between the GPS TEC and the NN predicted TEC values (Habarulema et al., 2009). The NN architecture that gave the least RMSE was adopted as the one suitable for TEC prediction. The computation of correlation coefficients has been used as a complementary option during the NN architecture determination. The RMSE and correlation coefficient, $\mu$ are defined as

$$
\begin{aligned}
& \text { RMSE }=\sqrt{\frac{1}{N} \sum_{i=1}^{N}\left(T^{m}-T^{p}\right)^{2}} \\
& \mu=\frac{1}{\delta_{m} \delta_{p}}\left(\sum_{i=1}^{N}\left(T_{i}^{m}-\bar{T}^{m}\right)\left(T_{i}^{p}-\bar{T}^{p}\right)\right)
\end{aligned}
$$

where $\delta_{m}$ and $\delta_{p}$ are the respective standard deviations for measured and predicted TEC defined by

$\delta_{m}=\sqrt{\frac{1}{N} \sum_{i=1}^{N}\left(T_{i}^{m}-\bar{T}^{m}\right)^{2}}$, 


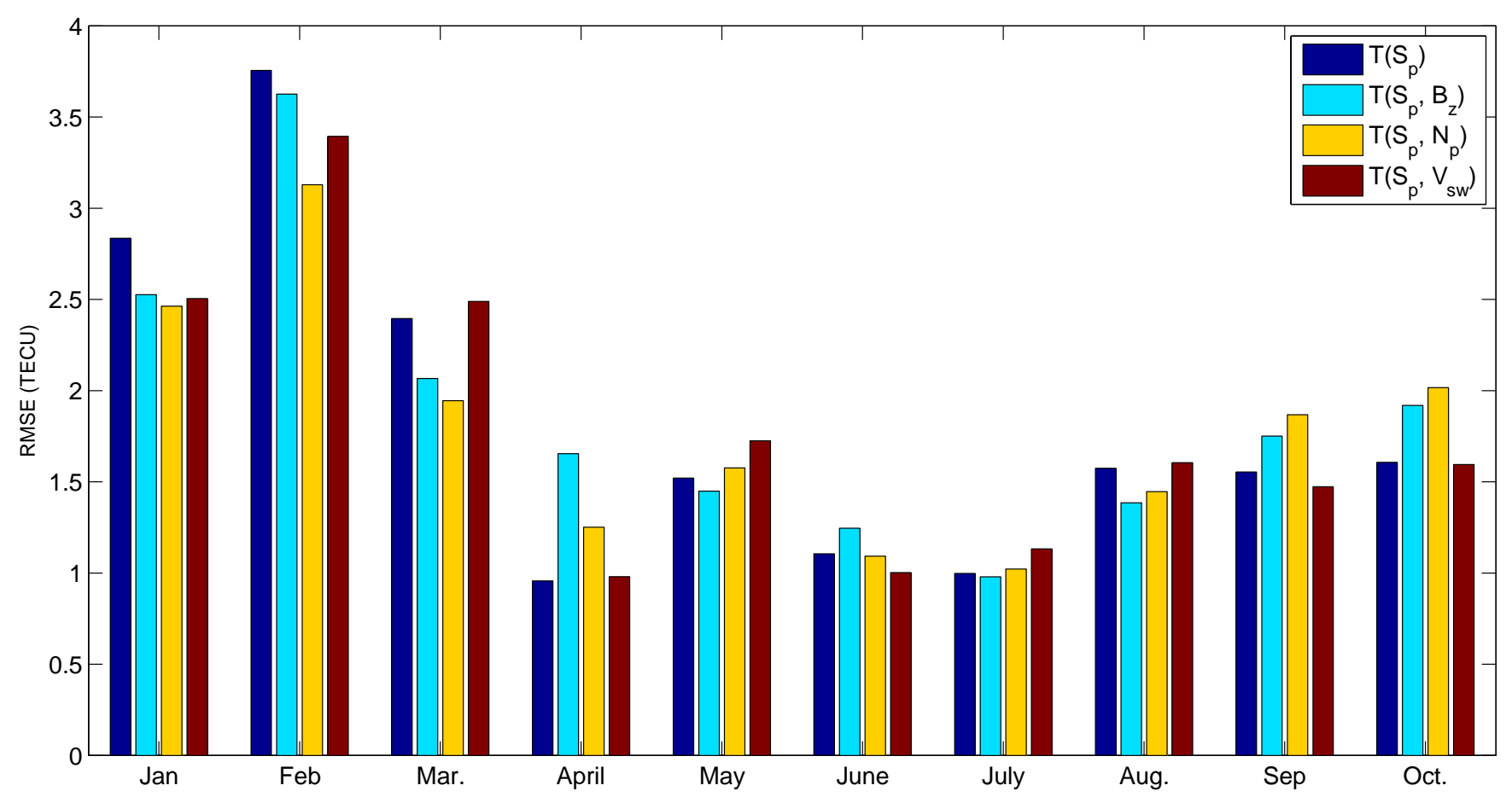

Fig. 11. Computed RMSE values between GPS TEC and predicted TEC for the available months in 2005.

$$
\delta_{p}=\sqrt{\frac{1}{N} \sum_{i=1}^{N}\left(T_{i}^{p}-\bar{T}^{p}\right)^{2}},
$$

and $T^{m}$ and $T^{p}$ are the measured and predicted TEC, $\bar{T}^{m}$ and $\bar{T}^{p}$ are their respective means over a length interval of the total number of observations, $N$ under consideration. Table 1 shows the RMSE values obtained for different network architectures on different days. The number of hidden nodes that provided the optimum architecture was found to be $\sim n+2$ where $\mathrm{n}$ is the number of inputs to the ENN. The final discussed results in the following section are obtained by predicting hourly and daily TEC as a function of either six or seven parameters and may be mathematically expressed as

$T_{p} \equiv\left\{\begin{array}{cc}f\left(S_{p}\right), & \text { for standard parameters } \\ f\left(S_{p}, V_{s w}\right), & V_{s w} \text { included } \\ f\left(S_{p}, N_{p}\right), & N_{p} \text { included } \\ f\left(S_{p}, B_{z}\right), & \text { IMF } B_{z} \text { included }\end{array}\right.$

where $S_{p} \equiv\{\mathrm{DN}, \mathrm{HR}, \mathrm{R} 4, \mathrm{~A} 8\}$ represent the standard parameters that influence TEC variability, $\mathrm{DN} \equiv\{\mathrm{DNC}, \mathrm{DNS}\}$ and $\mathrm{HR} \equiv\{\mathrm{HRC}, \mathrm{HRS}\} . \quad$ An important point to note is that we have used results from the ENN trained at Sutherland $\left(32.38^{\circ} \mathrm{S}, 20.81^{\circ} \mathrm{E}\right)$ to predict TEC changes at a receiver site located at Cape Town $\left(33.95^{\circ} \mathrm{S}, 18.47^{\circ} \mathrm{E}\right)$. Its basis is the recent study by Habarulema et al. (2009) which showed that it
Table 1. RMSE (between predicted and measured GPS TEC) for some days in 2005 for different architectures when DN, HR, R4 and $\mathrm{A} 8$ are included in the ENN as inputs.

\begin{tabular}{llrrrr}
\hline $\begin{array}{l}\text { Network } \\
\text { denotation }\end{array}$ & $\begin{array}{l}\text { Architecture } \\
\text { configuration }\end{array}$ & day 60 & day 100 & day 250 & day 291 \\
\hline A & $6: 6: 1$ & 13.3247 & 8.3594 & 5.8260 & 6.7112 \\
B & $6: 7: 1$ & 2.0740 & 2.3914 & 2.0723 & 2.4126 \\
C & $6: 8: 1$ & 2.1627 & 1.7717 & 1.3522 & 1.9618 \\
D & $6: 9: 1$ & 2.6417 & 3.2795 & 1.8668 & 1.9108 \\
E & $6: 10: 1$ & 2.8451 & 2.3629 & 1.8298 & 1.9035 \\
\hline
\end{tabular}

is possible to predict TEC variations at GPS receiver stations where data was not included in training, within a latitudinal range of $\sim 1-3$ degrees.

\section{Results and discussion}

\subsection{Diurnal TEC predictions}

Figures 3, 4, 5 and 6 show the comparison of hourly GPS TEC and the predicted values as a function of the standard parameters (seasonal and diurnal variations, solar and magnetic activities) and after separate inclusion of each solar wind parameter for days 58, 104, 185 and 285 in 2005 over Cape Town $\left(33.95^{\circ} \mathrm{S}, 18.47^{\circ} \mathrm{E}\right)$. Table 2 shows the computed RMSE values between GPS TEC and the corresponding predicted values. This table indicates that all solar wind 

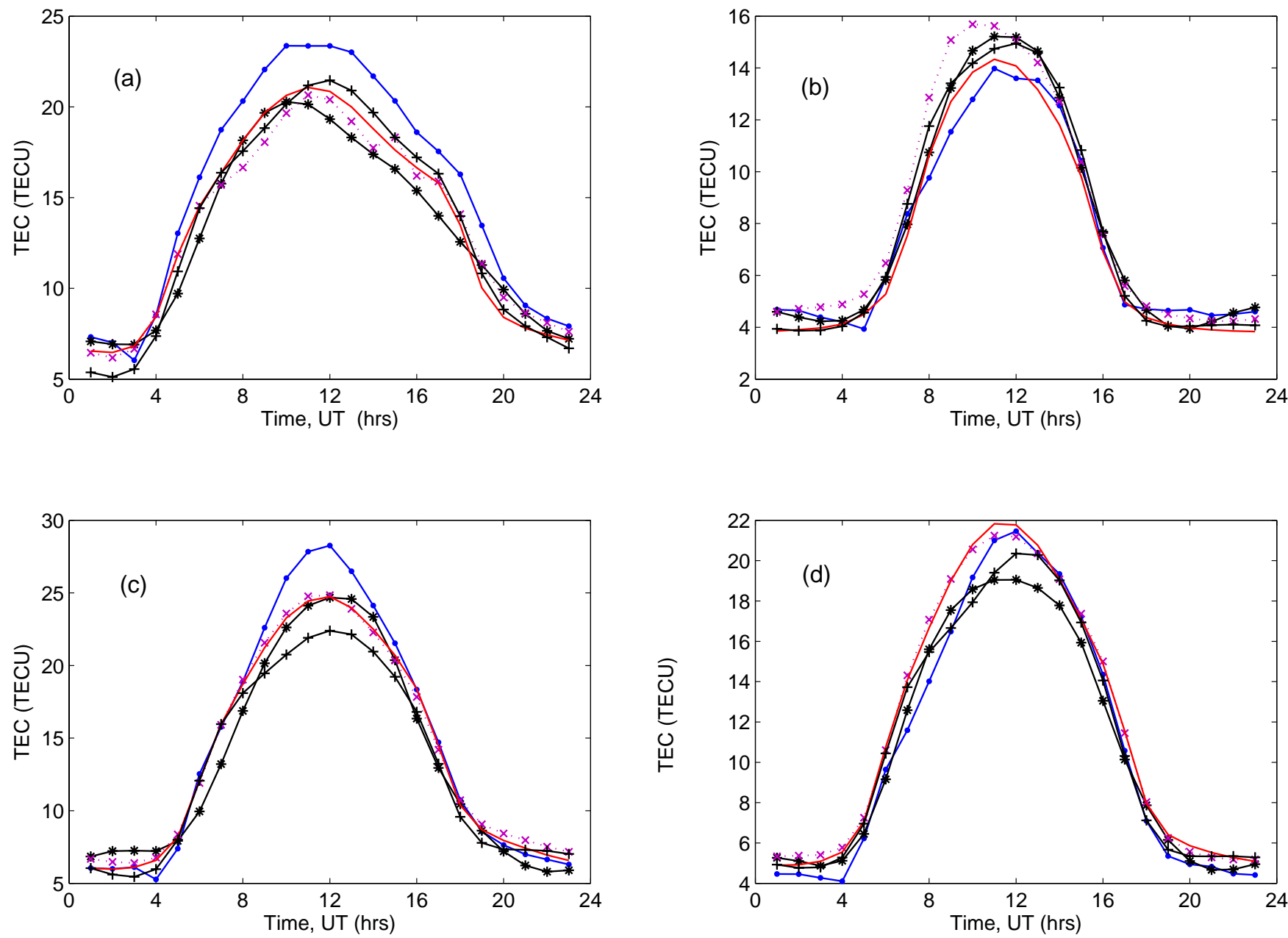

$$
\longrightarrow \text { GPS TEC } \rightarrow \text { * }-\mathrm{T}\left(\mathrm{S}_{\mathrm{p}}\right) \ldots \times \mathrm{T}\left(\mathrm{S}_{\mathrm{p}}, \mathrm{B}_{\mathrm{z}}\right)-\mathrm{T}\left(\mathrm{S}_{\mathrm{p}}, \mathrm{N}_{\mathrm{p}}\right) \multimap \mathrm{T}\left(\mathrm{S}_{\mathrm{p}}, \mathrm{V}_{\mathrm{sw}}\right)
$$

Fig. 12. A plot of monthly average GPS TEC for each hour and the corresponding predictions with all parameters used for the months representing (a) summer (January), (b) winter (June), (c) autumn (March) and (d) spring (September) in 2005.

Table 2. RMSE between GPS TEC and predicted TEC values for days 58, 104, 185 and 285 in 2005.

\begin{tabular}{lrrrr}
\hline Day & \multicolumn{4}{c}{ RMSE (TECU) between GPS TEC and } \\
(in 2005) & $T\left(S_{p}\right)$ & $\mathrm{T}\left(S_{p}, B_{z}\right)$ & $T\left(S_{p}, N_{p}\right)$ & $T\left(S_{p}, V_{s w}\right)$ \\
\hline 58 & 1.6171 & 1.5873 & 1.3105 & 1.3112 \\
104 & 2.3562 & 2.7691 & 2.7958 & 3.1242 \\
185 & 0.9472 & 0.9689 & 1.2377 & 1.2506 \\
285 & 1.2015 & 1.0100 & 0.9443 & 1.2727 \\
\hline
\end{tabular}

parameters considered slightly improved the prediction accuracy for day 58 while no improvement is observed on days 104 and 185. The inclusion of $B_{z}$ and $N_{p}$ gives improvements of $\sim 15.94 \%$ and $\sim 21.41 \%$ for day 285 while $V_{s w}$ slightly degrades the prediction accuracy by $\sim 5.93 \%$ with respect to $S_{p}$. Once again, these results are obtained by verifying the ENN at a GPS receiver site whose data was not involved in model development and this confirms the previous study by Habarulema et al. (2009). Prediction accuracies in terms of both degradation and improvement are close to the values of reference when solar wind parameters are included for these quiet days. In all cases, the TEC diurnal shape is correct at almost all times of the day.

Figures 7 and 8 show the variations of the solar wind parameters and GPS TEC along with the predicted values for 6 10 and 14-18 May 2005. These particular dates were chosen because they contained days when magnetic storms occured. These two figures are meant to specifically show the performance of the ENN model during prestorm, storm and post storm conditions. The prediction accuracies for these dates when different parameters are included in ENN modelling are shown in Fig. 9. There was a magnetic storm on 8 May 

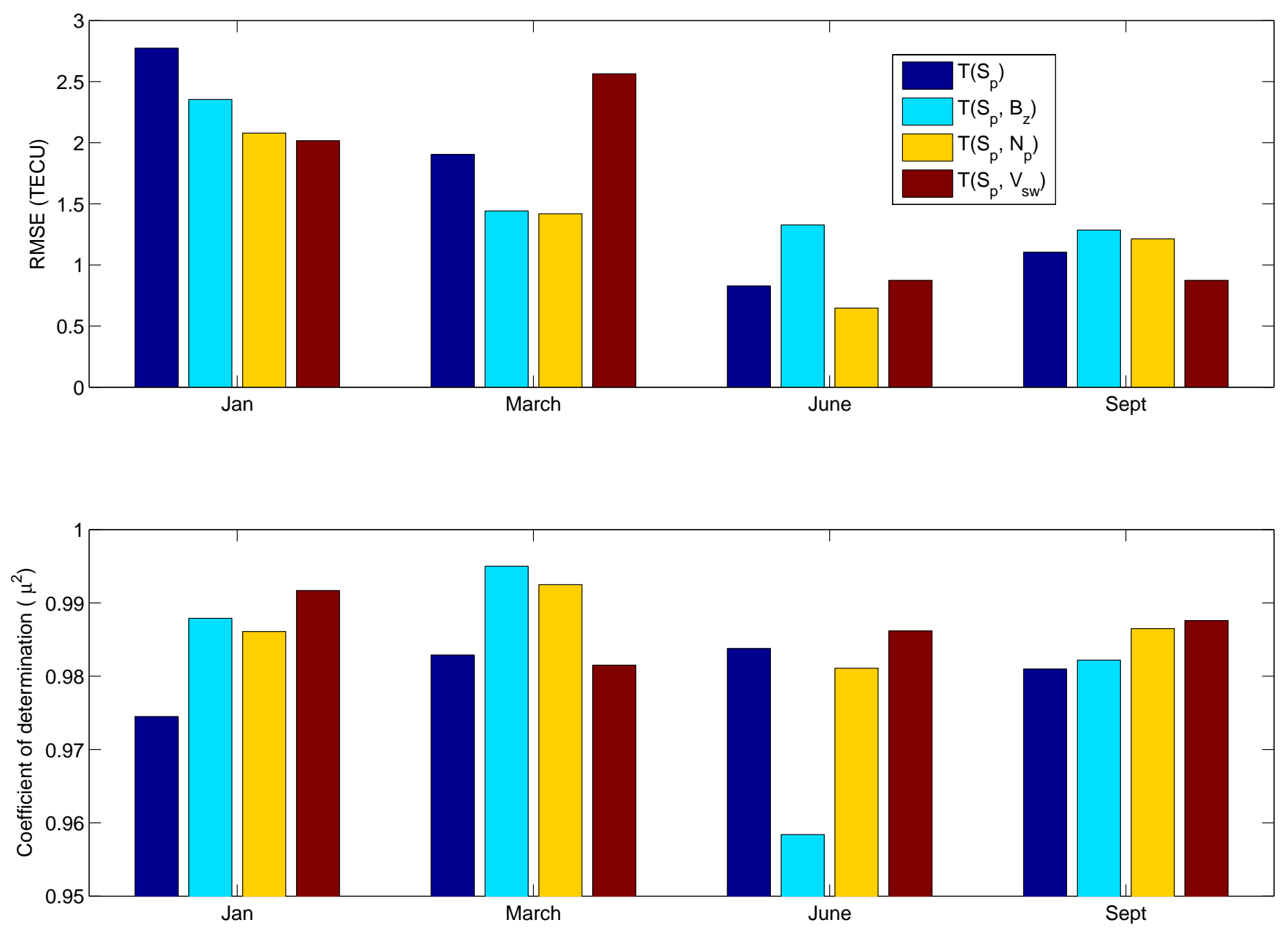

Fig. 13. Comparison of RMSE and coefficient of determination $\left(\mu^{2}\right)$ values for the months representing autumn, winter, spring and summer in 2005 .

which appears to have led to positive storm effects when the TEC values are compared to relatively quiet days before and after the storm. There was a stronger magnetic storm on 15 May 2005 that led to the significant fluctuation of IMF $B_{z}$ and sudden jump in both $V_{s w}$ and $N_{p}$. As a result significant TEC fluctuations are observed. For both of these storm days a statistical improvement is observed in Fig. 9 after the inclusion of $B_{z}$ and $N_{p}$. While the ENN correctly identifies the TEC variational pattern on 8 May, the result is different on 15 May for all parameters considered. In addition, all combinations of different parameters strongly underpredict the magnitude of TEC values on 8 May. In both cases, the inclusion of $V_{s w}$ degrades the prediction accuracy, an indication that solar wind velocity may not be an efficient representation of solar wind in TEC modelling during magnetic storms. This should be investigated further for more storm conditions. However, these results clearly demonstrate that it is still difficult to accurately predict diurnal TEC behaviour during magnetically disturbed conditions.

\subsection{Seasonal TEC predictions}

Figure 10 shows the comparison of average daily GPS TEC and the predicted values for January to October 2005 over Cape Town $\left(33.95^{\circ} \mathrm{S}, 18.47^{\circ} \mathrm{E}\right)$. Due to a lack of data, November and December are not represented. Figure 11 shows the computed RMSE values between GPS TEC and $T\left(S_{p}\right), T\left(S_{p}, B_{z}\right), T\left(S_{p}, N_{p}\right), T\left(S_{p}, V_{s w}\right)$. The three solar wind parameters included led to average improvements in TEC prediction for January and February. Both $B_{z}$ and $N_{p}$ improve the prediction accuracy for March and August while results are more or less constant for June and September for these solar wind parameters. No change is observed for October when $V_{s w}$ is included, while $B_{z}$ and $N_{p}$ degrade the prediction accuracy with respect to $S_{p}$. Figure 12 shows the average diurnal TEC variations for January, March, June and September representing summer, autumn, winter and spring, respectively. The distinct observation from this figure is that all parameters underpredicted GPS TEC for almost the whole month of January as seen in Fig. 12a 
except at 03:00 UT where $T\left(S_{p}\right), T\left(S_{p}, N_{p}\right)$ and $T\left(S_{p}, B_{z}\right)$ are greater than GPS TEC by $\sim 0.870$ TECU, 0.779 TECU and 0.629 TECU, respectively, and 04:00 UT where $T\left(S_{p}\right.$, $\left.N_{p}\right) \simeq T\left(S_{p}, B_{z}\right) \simeq$ GPS TEC. An underprediction is also observed for March between $\sim 08: 00$ UT and 16:00 UT. Figure 13 is a representation of RMSE between derived GPS TEC and its predicted values as a function of all considered parameters. Represented in Fig. 13 are also the coefficients of determination (square of correlation coefficients) between GPS TEC and TEC values as predicted by different parameter combinations. With regard to $S_{p}$ as a benchmark, $B_{z}, N_{p}$ and $V_{s w}$ improve the TEC predictions by $\sim 15.16 \%$, $\sim 25.07 \%$ and $\sim 27.38 \%$, respecively for January (summer). For March, $B_{z}$ and $N_{p}$ gave improvements of $\sim 24.27 \%$ and $\sim 25.50 \%$, respectively. In June (winter), the inclusion of $V_{s w}$ doesn't change results while $N_{p}$ improves the prediction by an average of $\sim 21.86 \%$. For September, both $B_{z}$ and $N_{p}$ led to an overprediction between $\sim 05: 00 \mathrm{UT}$ and 13:00 UT, while $S_{p}$ underpredicts TEC between 09:00 UT and 11:00 UT. Difficulties in generating accurate predictions in spring have been observed before (Habarulema et al., 2007) and may be due to the strong variations exhibited by TEC during the equinoxes (Kouris et al., 1999) and therefore all parameters that influence TEC probably have high variation levels (Habarulema et al., 2009) including solar wind. In the Northern Hemisphere, Girish et al. (1997) obtained a significant correllation between the variations of solar wind parameters and TEC during the sunspot maximum in winter and spring. We have obtained a statistical correllation between TEC variations and atleast one of solar wind parameters investigated in all seasons through ENN modelling. Our results indicate a relative agreement for $N_{p}$ and $V_{s w}$ during the declining phase of the solar cycle 23 (year 2005) in the Southern Hemisphere which may be a confirmation that the solar wind dynamics influence TEC variations at both solar activity levels and in both hemispheres. Based on statistical analysis, it can be deduced that both daily and monthly average TEC predictions were improved by including $V_{s w}$ in the database for spring. Confirmation of this result requires more analysis since only one station's data has been analysed.

\section{Conclusions and future work}

This paper has attempted to explain the study of quantifying solar wind effects on GPS TEC using ENN modelling. GPS TEC has been predicted as a function of DN, HR, R4 and A8, and the results used as a benchmark to try and quantify solar wind influence on GPS TEC. Statistical analysis revealed both improvements and degradation in TEC prediction accuracies for different parameter combinations and for different days and seasons. An analysis was performed for both quiet and storm conditions. Although the inclusion of $B_{z}$ and $N_{p}$ improved the prediction accuracy for both stormy days ( 8 and 15 May), an underprediction was observed for 8 May while the variational trend pattern was incorrect for 15 May. These results show that it is still difficult to predict TEC variability during magnetic storms. We have investigated only three solar wind parameters in this study and although a degradation in TEC prediction has been observed in some cases, results indicate that the solar wind parameter may be a potential additional input to the ENN during TEC predictions. This claim should be investigaed further by; (1) examining more solar wind parameters, (2) looking for a method of combining the three investigated parameters to obtain one single quantity containing $B_{z}, N_{p}$ and $V_{s w}$ information since they all provide varying magnitudes in the prediction accuracy improvement during different days and seasons and (3) including the solar wind parameters in a multi-station model where the geographical location of the GPS receivers will be taken into account.

Acknowledgements. Thanks to Chief Directorate Surveys and Mappings (CDSM) and IGS community for making GPS data available. The authors acknowledge the ACE project teams (D. J. McComas of Southwest Research Institute and N. Ness of Bartol Research Institute) for making the solar wind data available. J. B. Habarulema greatly thanks the South African National Astrophysics and Space Science Programme, University of Cape Town, South Africa for the financial support.

Topical Editor M. Pinnock thanks two anonymous referees for their help in evaluating this paper.

\section{References}

Biktash, L. Z., Maruyama, T., and Nozaki, K.: The solar wind control of the equatorial ionosphere dynamics during geomagnetic storms, Adv. Space Res., 41, 562-568, 2008.

Bishop, C. M.: Neural Networks for Pattern Recognition, Oxford University Press Inc., New York, 1995.

Bodén, M.: A guide to recurrent neural networks and backpropagation, in: In the Dallas project, SICS Technical Report T2002:03, SICS, 2002.

Buonsanto, M. J.: Ionospheric storms: A review, Space Sci. Rev., 88, 563-601, 1999.

Elman, J. L.: Finding Structure in Time, Cognitive Science, 14, 179-211, 1990.

Fausett, L.: Fundamentals of Neural Networks; Architectures, Algorithms and Applications, Prentice-Hall, Inc. New Jersey, 07632, 1994.

Fedrizzi, M., de Paula, E. R., Langley, R. B., Komjathy, A., Batista, I. S., and Kantor, I.: Study of the March 31, 2001 magnetic storm effects on the ionosphere using GPS data, Adv. Space Res., 36, 534-545, 2005.

Girish, T. E., Jayachandran, B., and Shamsudeen, S. P.: Influence of solar wind on the TEC variations at mid and subauroral latitudes during sunspot maximum, Acta geod. Geoph. Hung., 32(3-4), 287-292, 1997.

Habarulema, J. B.: A feasibility study into Total Electron Content prediction using Neural Networks, Master's thesis, Rhodes University, Grahamstown, South Africa, 2007.

Habarulema, J. B., McKinnell, L. A., and Cilliers, P. J.: Prediction of global positioning system total electron content using neu- 
ral networks over South Africa, J. Atmos. Solar Terr. Phys., 69, 1842-1850, 2007.

Habarulema, J. B., McKinnell, L.-A., Cilliers, P. J., and Opperman, B. D. L.: Application of neural networks to South African GPS TEC modelling, Adv. Space Res., in press, doi:10.1016/j.asr.2008.08.020, 2009.

Haykin, S.: Neural Networks, A Comprehensive Foundation, Macmillan College Publishing Company, 1994.

Hernandez-Pajares, M., Juan, J., and Sanz, J.: Neural network modelling of the ionospheric electron content at global scale using GPS, Radio Sci., 32, 1081-1090, 1997.

Hofmann-Wellenhof, B., Lichtenegger, H., and Collins, J.: Global Positioning System Theory and Practice, Springer-Verlag Wien New York, 1992.

Jakowski, N., Hocke, K., Schluter, S., and Heise, S.: Space weather effects detected by GPS based TEC monitoring, in: Workshop on Space Weather, WPP-155, ESTEC, Noordwijk,, pp. 241-244, 1999.

Kouris, S. S., Fotiadis, D. N., and Zolesi, B.: Specifications of the Fregion variations for quiet and disturbed conditions, Phys. Chem. Earth, 24, 1999.

Langley, R. B.: GPS, the Ionosphere, and the Solar Maximum, GPS World, 11, 44-49, 2000.

Li, G., Ning, B., Zhao, B., Liu, L., Liu, J. Y., and Yumoto, K.: Effects of geomagnetic storms on GPS ionospheric scintillations at Sanya, J. Atmos. Solar-Terr. Phys., 70(7), 1034-1045, 2008.

Lundestedt, H.: Solar activity modelled and forecasted: A new approach, Adv. Space Res., 38, 862-867, 2006.

Lyon, J. G.: The Solar Wind-Magnetosphere-Ionosphere System, Science Rev., 288, 1987-1991, 2000.

Marra, S. and Marabito, F. C.: A New Technique for Solar Activity Forecasting using Recurrent Elman Networks, Int. J. Cognitive Intelligence, 3, 8-13, 2005.
Meza, A., Zele, M. A. V., Brunini, C., and Cabassi, I. R.: Vertical total electron content and geomagnetic pertubations at midand sub-auroral southern latitudes during geomagnetic storms, J. Atmos. Solar Terr. Phys., 67, 315-323, 2005.

Opperman, B.: Reconstructing Ionospheric TEC over South Africa using signals from a Regional GPS network, PhD thesis Rhodes University, Grahamstown, South Africa, 2007.

Opperman, B. D. L., Cilliers, P. J., McKinnell, L. A., and Haggard, R.: Development of a regional GPS-based ionospheric TEC model for South Africa, Adv. Space Res., 39, 808-815, 2007.

Poole, A. W. and McKinnell, L.-A.: On the predictability of foF2 using neural networks, Radio Sci., 1, 225-234, 2000.

Sethia, G., Deshpande, M. R., and Rastogi, R. G.: The solar wind influences plasmasphere electron content, Nature, 276, 482 , doi:10.1038/276482a0, 1978.

Stankov, S. M., Kutiev, I., Jakowski, N., and Wehrenpfennig, A.: A new method for total electron content forecasting using Global Positioning System measurements, in: Proc. ESA Space Weather Workshop, Noordwijk, The Netherlands, pp. 169-172, 2001.

Tulunay, E., Senalp, E., Radicella, S., and Tulunay, Y.: Forecasting total electron content maps by neural network technique, Radio Sci., 41, doi:10.1029/2005RS003285, 2006.

Vandegriff, J., Wagstaff, K., and Ho, G. J. P.: Forecasting space weather: Predicting interplanetary shocks using neural networks, Adv. Space Res., 36, 2323-2327, 2005.

Weigel, R. S., Vassiliadis, D., and Klimas, A. J.: Coupling of the solar wind to temporal fluctuations in ground magnetic fields, Geophys. Res. Lett., 29, 19, doi:10.1029/2002GL014740, 2002.

Weigel, R. S., Klimas, A. J., and Vassiliadis, D.: Solar wind coupling to and predictability of ground magnetic fields and their time derivatives, J. Geophys. Res., 108(A7), 1298, doi:10.1029/2002JA009627, 2003.

Wu, J.-G. and Lundstedt, H.: Neural network modeling of solar wind-magnetosphere interaction, J. Geophys. Res., 102(A7), 14457-14466, 1997. 